\title{
Mind the Brain: The mediating and moderating role of neurophysiology
}

\author{
Siobhán Harty, Francesco Sella, \& Roi Cohen Kadosh \\ Department of Experimental Psychology, University of Oxford, Oxford OX1 3UD, United Kingdom \\ E-mail Addresses: siobhan.harty@psy.ox.ac.uk; francesco.sella@psy.ox.ac.uk; \\ roi.cohenkadosh@psy.ox.ac.uk
}

\begin{abstract}
Summary
Most studies involving experimental manipulations or interventions tailored to modulate behavior do not account for variability in the critical antecedent of behavior, the brain. Here, we describe elegant approaches to model the role that neurophysiology can play in mediating or moderating relationships in this context. We highlight the capacity for these approaches to improve the inferential power of research, bridge the gap between neural and behavioral levels of analysis, and bolster the prospects for reproducibility.
\end{abstract}

\section{Acknowledgements}

This work was supported by grants from the James S. McDonnell Foundation 21st Century Science Initiative in Understanding Human Cognition and the European Research Council (Learning and Achievement; 338065). 
Most basic and translational research in the cognitive sciences entails manipulating independent variables with the view to observing the effect of these manipulations on behavioral outcomes of interest. When designing such research and analysing the resulting data we must be cognisant of the multifaceted systems from which the data are drawn and endeavor to account for variables that may modify or inform the relationship between our independent variables and outcomes of interest. Factors such as gender, age, socioeconomic status and level of education constitute a few of the factors that are regularly taken into account in the literature. However, of particular pertinence in this field is the fact that the brain, the antecedent to all nonreflexive behavior, is an exquisitely complex and highly dynamic system that will exhibit both intra- and inter-individual differences even in response to tightly controlled experimental stimuli. This intervening role of the brain is frequently overlooked in studies.

In this article, we describe some practical and elegant statistical frameworks which have been remarkably underutilized by many cognitive scientists to date, namely mediation and moderation analyses. We highlight how the inclusion of theoretically implicated neurophysiological indices in these statistical frameworks provides a powerful means for bridging the gap between neural and behavioral levels of analysis, and improving the prospects for reproducibility in the cognitive sciences. We use research scenarios concerning experimental manipulations tailored to modulate behavior as a model to explain how the neurophysiological milieu can mediate or moderate behavioral effects. However, from these examples it should be apparent that there is great scope for exploiting these frameworks in other areas of cognitive science.

Mediation analysis is a regression approach wherein the goal is to establish the indirect effect of independent variables on an outcome variable through a mediating variable. In the simplest version of this statistical model, an experimental manipulation (Condition A versus Condition B; Treatment vs. Placebo) could constitute the independent variable. A theoretically implicated cognitive process or neurophysiological index could constitute the mediating variable, and a behavioral index of interest could constitute the outcome variable.

The first step of a mediation analysis involves determining whether there is a significant difference in the behavior of interest for Condition A versus B (c path), as indexed by a linear regression. This identifies the direct effect of the manipulation on behavior, and it is common practice to solely focus on this bivariate relationship. However, in mediation analysis (Figure 1A), a second step investigates whether there is a differential effect of Condition A versus B on the mediating variable (a path). A significant relationship here implies that the manipulation had an effect on the proposed mediating variable. Next, the relationship between the mediating variable and behavioral index is examined, while simultaneously accounting for the impact of the experimental manipulation ( $b$ path). Finally, the mediation hypothesis is evaluated, and the manner by which one tests this should be guided by the sample size (see Fritz \& MacKinnon, 2007; Figure 1A). If a mediation effect is established, it can be claimed that the proposed mediating variable mediates the relationship between the experimental manipulation and behavior. Importantly, a mediation effect can be identified irrespective of an initial significant association between the experimental manipulation and the outcome (Hayes, 2009). Accordingly, mediation analyses can disclose associations between independent and outcome variables that may be obscured solely focussing on averaged data or bivariate relationships.

This approach has widespread applications but it is particularly valuable for research concerning interventions such as cognitive training, pharmacological manipulations, or brain stimulation. Within this research sphere the effectiveness of an intervention for modulating behavior is predominantly evaluated by analysing the direct effect of the intervention (Treatment versus Placebo) on the 
behavior of interest. A major limitation of this approach is that it disregards the fact that the impact of an intervention on behavioral outcomes will inevitably depend on how the neurophysiological milieu of each individual responds to the intervention. Consider, for example, a scenario whereby one is interested in determining the effects of non-invasive brain stimulation (NIBS) over dorsolateral prefrontal cortex (dlPFC), on arithmetic learning, which putatively relies on dlPFC functioning (Snowball et al., 2013). If we quantify the intervention-related response of the dlPFC with a neurophysiological assay, we can verify the validity of assumptions about the involvement of the dIPFC in arithmetic learning, the effect of NIBS on activity in this area, and the extent to which the pattern of effects are consistent across all participants. Furthermore, the inclusion of the interventionrelated response of the dIPFC as the proposed mediating variable in a mediation model would proffer nuanced insights on the determinants of the behavioral effects, or lack thereof. Importantly, with this approach, if one fails to observe the hypothesised effect on behavior, there is scope for arbitrating between different explanations by verifying whether all, some, or none, of the above-mentioned assumptions were met. The systematic assessment of theoretically implicated brain indices and their inclusion in a mediation model may thus reduce the chances of spurious conclusions and provide a more comprehensive understanding of the dynamic interaction between experimental manipulations, brain and behavior.

\section{Insert Figure 1 here}

Moderation analysis is also a form of regression analysis, but here the aim is to determine whether the relationships between independent and dependent variables change as a function of a third variable (i.e., statistical interaction), known as a moderator. In the context of the above research scenario, let us assume that the effect of NIBS on arithmetic learning depends upon the natural spontaneous functioning of the dlPFC (Figure 1B). In this case, moderation analyses can provide a powerful unified framework for demonstrating that the relationship between NIBS and behavior changes for different levels of functioning in the targeted region. Hence, similar to mediation analyses, this approach can facilitate a more refined interpretation of results, and can accordingly provide information to guide the development of more personalised and effective intervention protocols.

Building upon these two frameworks, one can generate more complex hypotheses where mediation and moderation co-occur. For instance, a mediation effect may be contingent on the level of a moderator, or a moderation effect may be carried through a mediating variable. Such scenarios can be modelled using moderated-mediation and mediated-moderation analyses, respectively (see Preacher, Rucker, \& Hayes, 2007).

In summary, the predominant approach of using bivariate analyses for evaluating the relationship between experimental manipulations and behavioral outcomes can veil important information and lead to unreliable inferences. Instead of simply seeking a dichotomous answer about whether the experimental manipulation works we should endeavor to design our studies and analyse our data in ways that can address questions about why, how, and for whom the experimental manipulation is effective. We suggest that the inclusion of theoretically implicated neurophysiological indices in mediation and moderation models constitute valuable approaches for progressing the field in this direction. These approaches have the capacity to enhance the inferential power of research by revealing important information about implicated mechanisms and the dynamic interaction between 
experimental manipulations, brain and behavior. Exploiting these approaches should also yield information for guiding the design of more effective and personalised cognitive interventions. And finally, the nuanced insights that these approaches afford should reduce the likelihood of type 1 and type 2 errors, and accordingly bolster the prospects for reproducibility in the field.

\section{Box 1. Open Source Plug-ins for Mediation and Moderation Analyses.}

We direct readers to Andrew Hayes' website at the following address: http://afhayes.com/

Here one can download open source documentation and plug-ins for SPSS and SAS which provide suites of analysis templates for mediation and moderation analyses. These allow for varying levels of complexity, including frameworks for moderated-mediation and mediated-moderation, in addition to options for incorporating multiple mediators, moderators and covariates.

Mediation and moderation analyses can also be run using packages for the open source statistical software R (e.g., The Lavaan package; Rosseel, 2012).

\section{Figure legend}

Figure 1. Schematics of mediation (A) and moderation (B) analyses: A) Upper panel: A linear regression examining the relationship between the independent variable (Group) and the outcome behavioral measure (c path). One can proceed to the analyses in the lower panel irrespective of whether a significant relationship is observed here. Lower panel: The effect of the independent variable on the neurophysiological index is evaluated with a linear regression (a path). The relationship between the neurophysiological index and the outcome variable is evaluated with another linear regression, wherein the independent variable is also included as predictor ( $b$ path). The effect of the independent variable on the outcome variable is re-evaluated using a linear regression that also includes the neurophysiological mediator ( $c^{\prime}$ path). The three most common approaches to conclude a mediation effect are: 1) using bootstrapping with replacement to obtain a distribution of the product of the a path and $b$ path regression coefficients, and establish whether the $95 \%$ confidence intervals of the distribution overlap with zero (Hayes, 2013; Mackinnon \& Fairchild, 2009); 2) verifying that both the a path and the $b$ path are statistically significant (test of joint significance; Kenny, Kashy, \& Bolger, 1998) ; or 3) the product of the regression coefficients from the a path and $b$ path is significantly different from zero when evaluated using the Sobel test (Sobel, 1986); B) Upper panel: A linear regression examining the relationship between the independent variable (Group) and the outcome behavioral measure (c path). Lower panel: In accordance with standard convention for moderation analyses (Aiken and West, 1991), the estimated value of the outcome variable for each group is reported at the mean, one standard deviation below the mean and one standard deviation above the mean, of the proposed moderating variable. This example shows a significant moderation effect: the impact of the experimental manipulation on the outcome variable changes according to the value of the neurophysiological index (i.e. the moderator). 


\section{References}

Aiken, L. S. \& West, S. G. (1991), Multiple Regression: Testing and Interpreting Interactions, Thousand Oaks, CA: Sage.

Fritz, M. S., \& MacKinnon, D. P. (2007). Required sample size to detect the mediated effect. Psychological Science, 18(3), 233-239. http://doi.org/10.1111/j.1467-9280.2007.01882.x

Hayes, A. F. (2009). Beyond Baron and Kenny: Statistical Mediation Analysis in the New Millennium. Communication Monographs, 76(4), 408-420. http://doi.org/10.1080/03637750903310360

Hayes, A. F. (2013). Introduction to mediation, moderation, and conditional process analysis. New York, NY: Guilford, 3-4. http://doi.org/978-1-60918-230-4

Kenny, D. A., Kashy, D., \& Bolger, N. (1998). Data analysis in social psychology. Handbook of Social Psychology, 233-265. http://doi.org/10.1002/pits.10035

Mackinnon, D. P., \& Fairchild, A. J. (2009). Current Directions in Mediation Analysis. Current Directions in Psychological Science: A Journal of the American Psychological Society, 18(1), 16. http://doi.org/10.1111/j.1467-8721.2009.01598.x

Preacher, K. J., Rucker, D. D., \& Hayes, A. F. (2007). Addressing moderated mediation hypotheses: theory, methods, and prescriptions. Multivariate Behavioral Research, 42(1), 185-227. http://doi.org/10.1080/00273170701341316

Rosseel, Y. (2012). lavaan: An R Package for Structural Equation Modeling. Journal of Statistical Software, 48(2), 1-36.

Snowball, A., Tachtsidis, I., Popescu, T., Thompson, J., Delazer, M., Zamarian, L., ... Cohen Kadosh, R. (2013). Long-term enhancement of brain function and cognition using cognitive training and brain stimulation. Current Biology: $C B, 23(11), 987-92$. http://doi.org/10.1016/j.cub.2013.04.045

Sobel, M. E. (1986). Some New Results on Indirect Effects and Their Standard Errors in Covariance Structure Models. Sociological Methodology, 16(16), 159-186. 
Figure 
A)
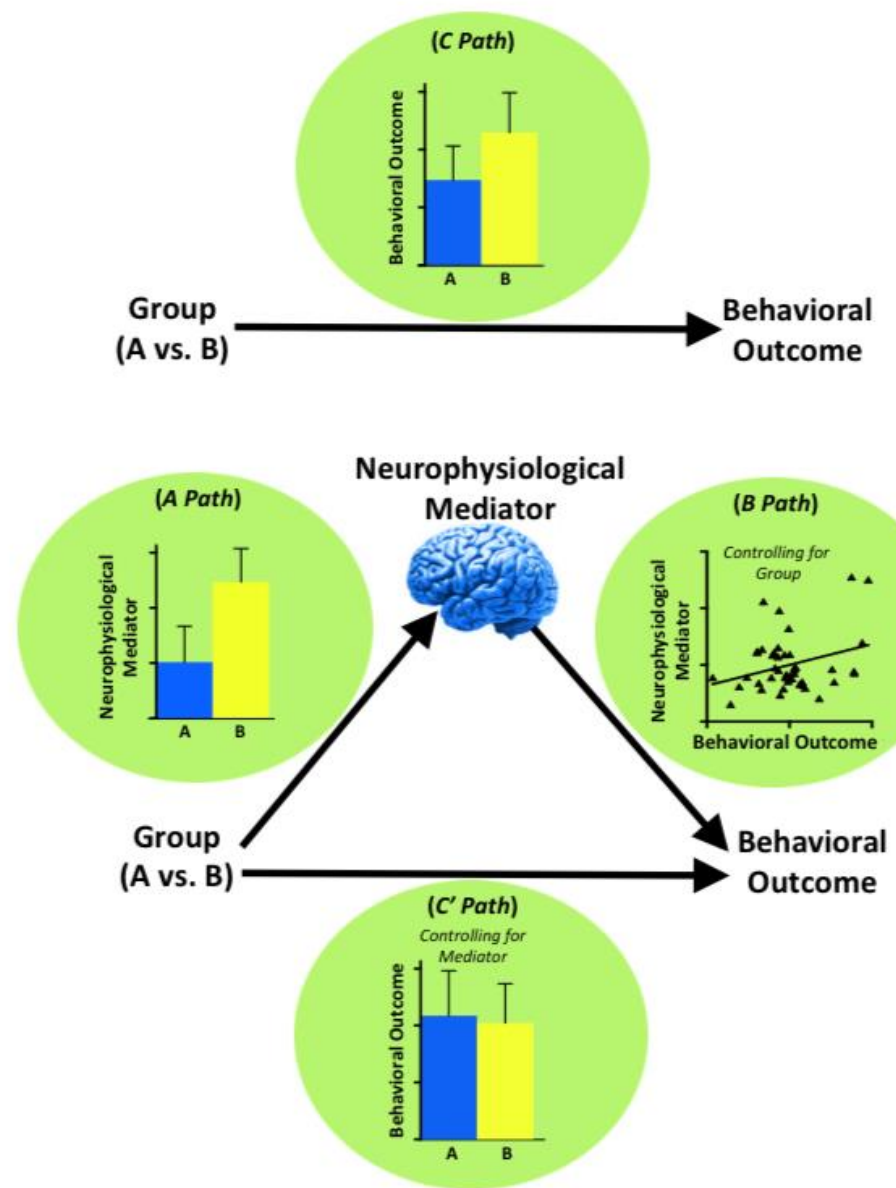

B)

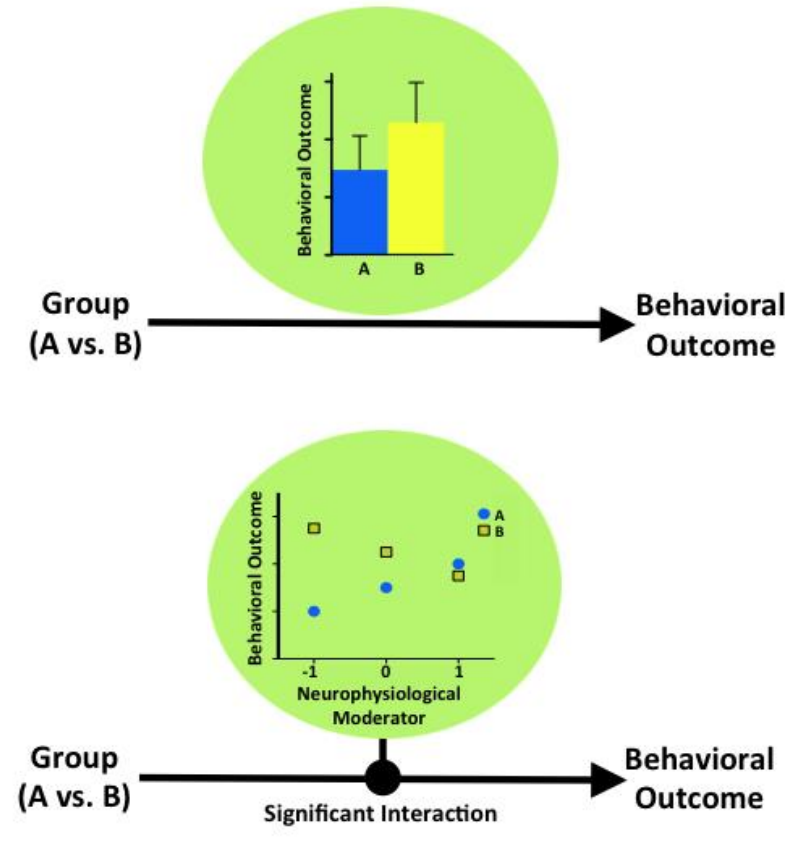

